\title{
Enhanced Mechanical Damping in Electrospun Polymer Fibers with Liquid Cores: Applications to Sound Damping
}

\author{
Michael J. Bertocchi ${ }^{\dagger+}$, Pearl Vang ${ }^{\dagger}$, Robert B. Balow ${ }^{\dagger}$, James H. Wynne ${ }^{\dagger}$, Jeffrey G. Lundin ${ }^{\dagger *}$ \\ $\dagger$ Chemistry Division, U.S. Naval Research Laboratory, \\ 4555 Overlook Avenue SW, Washington, D.C. 20375, USA \\ American Society for Engineering Education Postdoctoral Fellow \\ 1818 N Street, N.W. Suite 600, Washington DC 20036-2479, USA
}

*Correspondence: jeffrey.lundin@nrl.navy.mil 


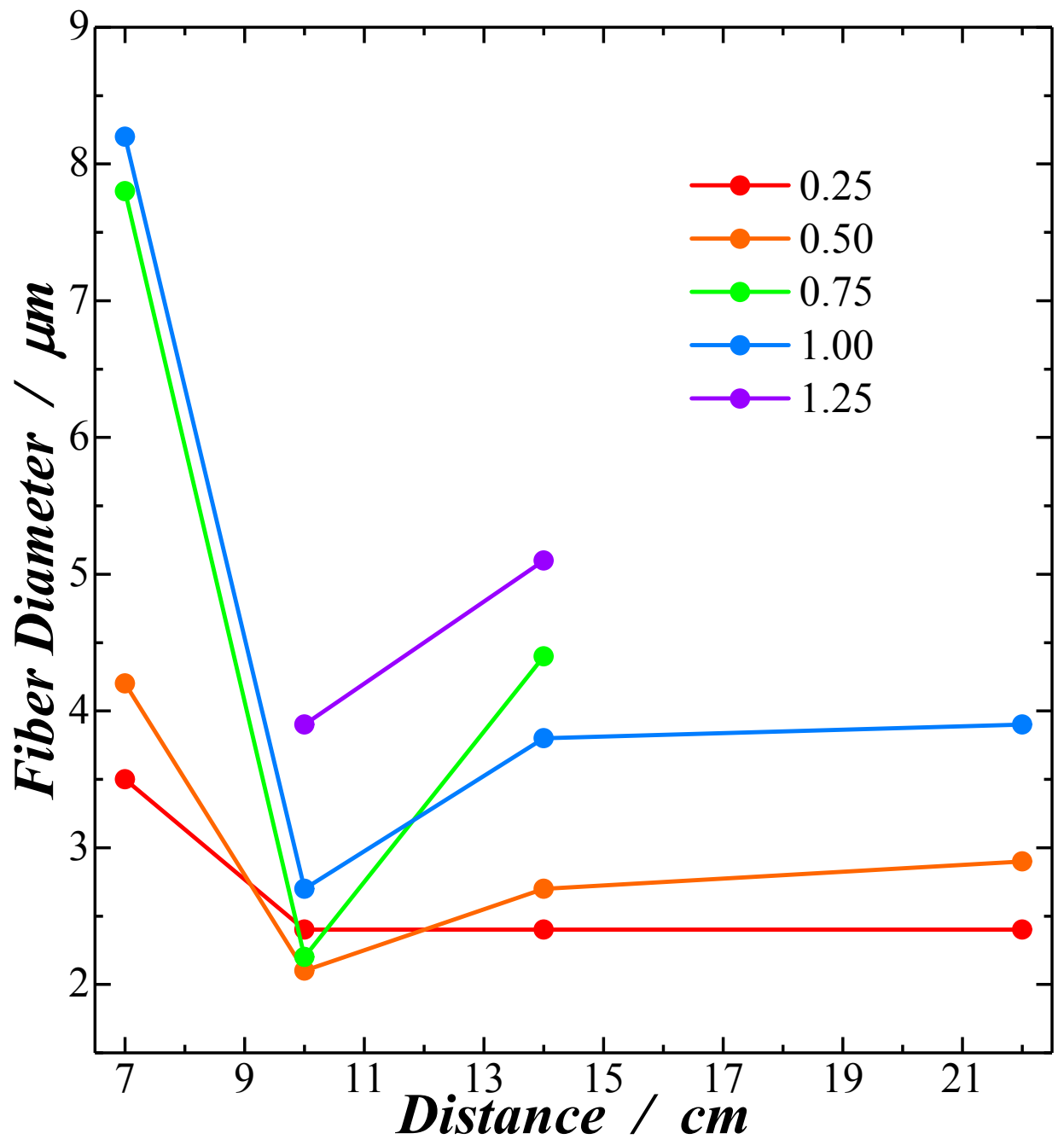

Figure S1. Plot of fiber diameter as a function of collector-to-spinneret separation distance at different flow rates of PEG200. The flow rate of the polymer sheath solution was $3 \mathrm{~mL} \mathrm{hr}^{-1}$. 


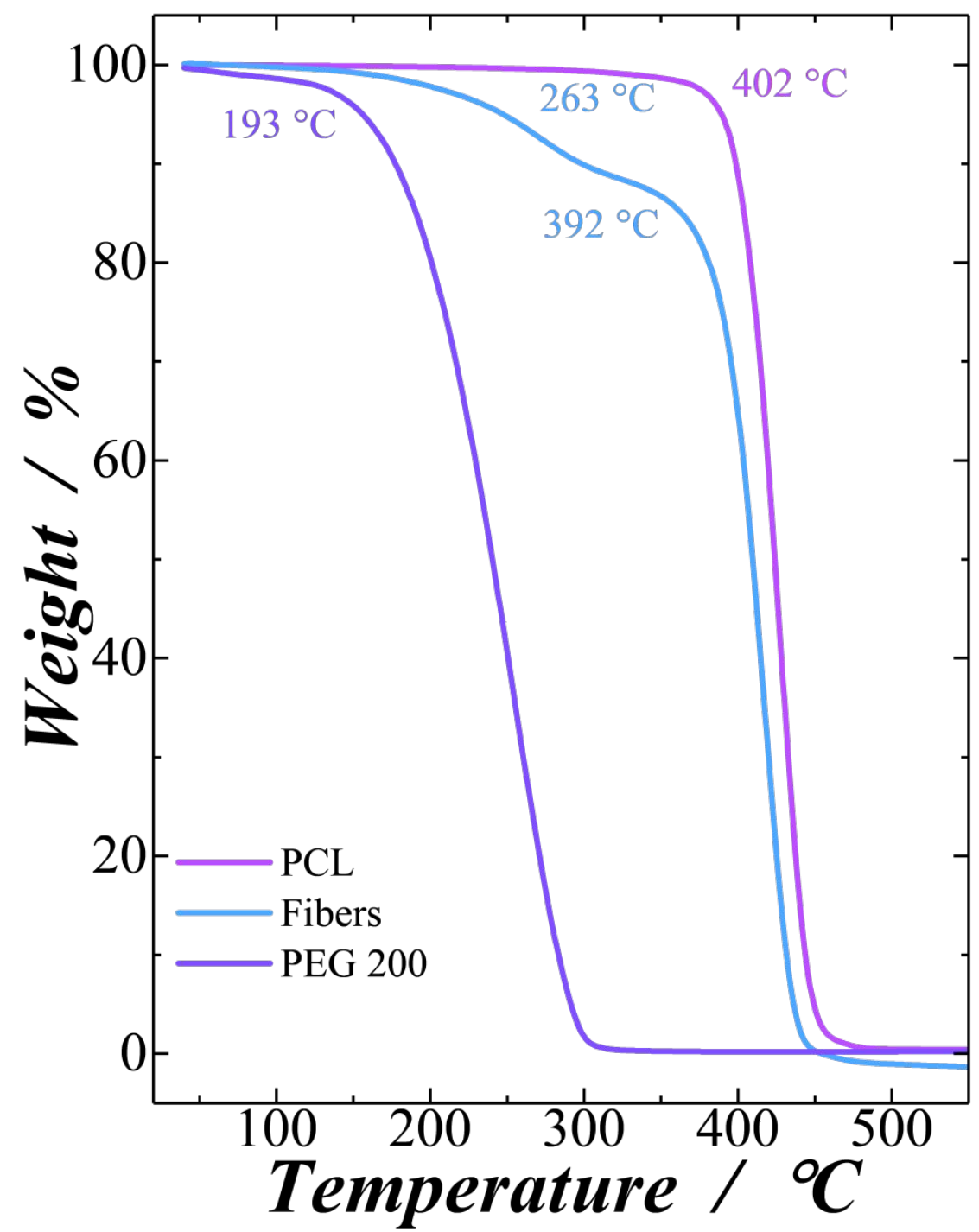

Figure S2. Thermogravimetric analyses of PCL, PEG200, and PCL-PEG200 electrospun fibers. Scans were collected at a heating rate of $10^{\circ} \mathrm{C} \mathrm{min}{ }^{-1}$. 


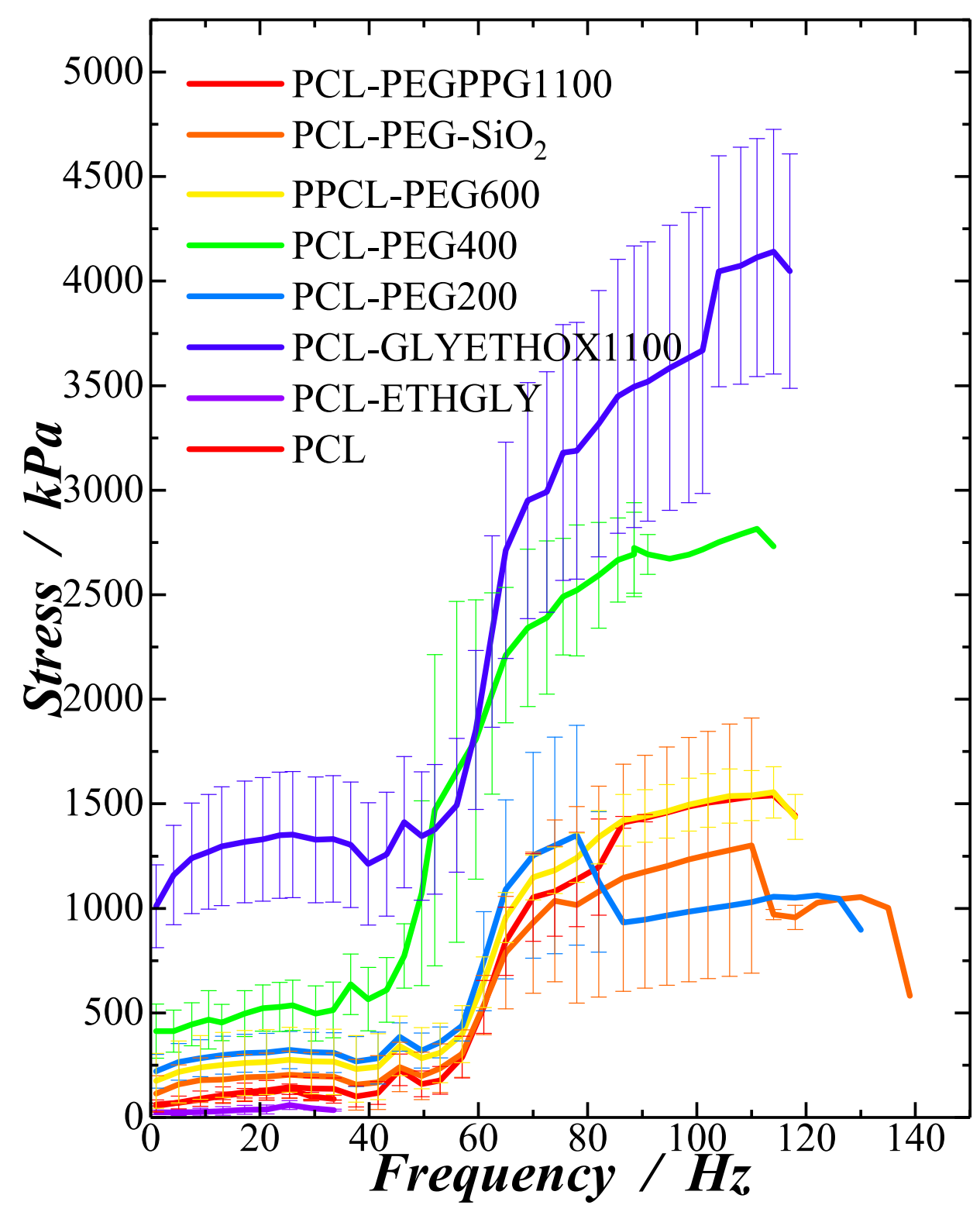

Figure S3. Stress curves of the electrospun fiber mats and a PCL film as a function of mechanical oscillation in the tensile mode at $1 \%$ stain. The curves represent the average of three measurements and the error bars denote the standard deviation. 

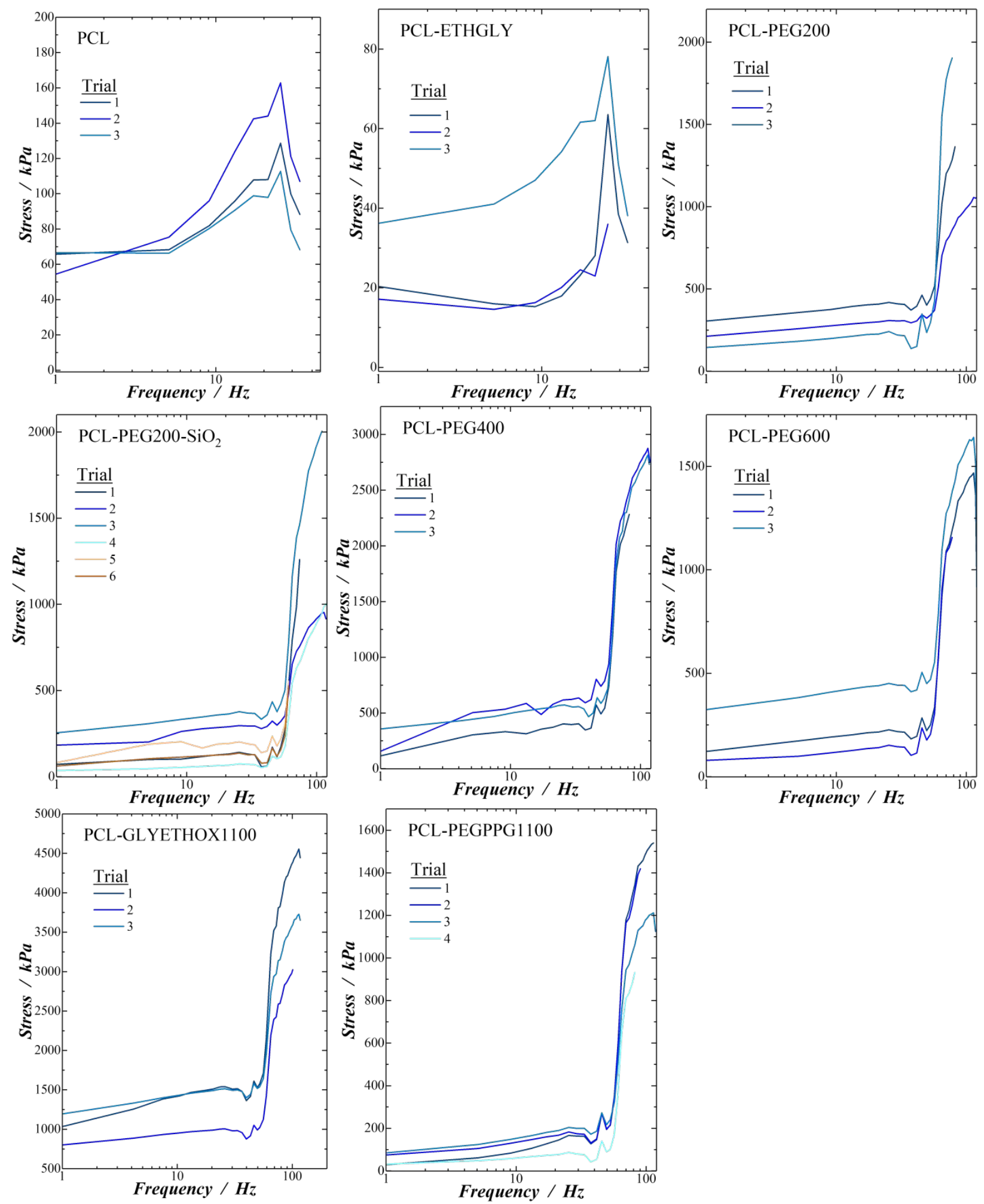

Figure S4. Individual stress curves of electrospun fiber mats as a function of mechanical oscillation in the tensile mode at $1 \%$ stain. 


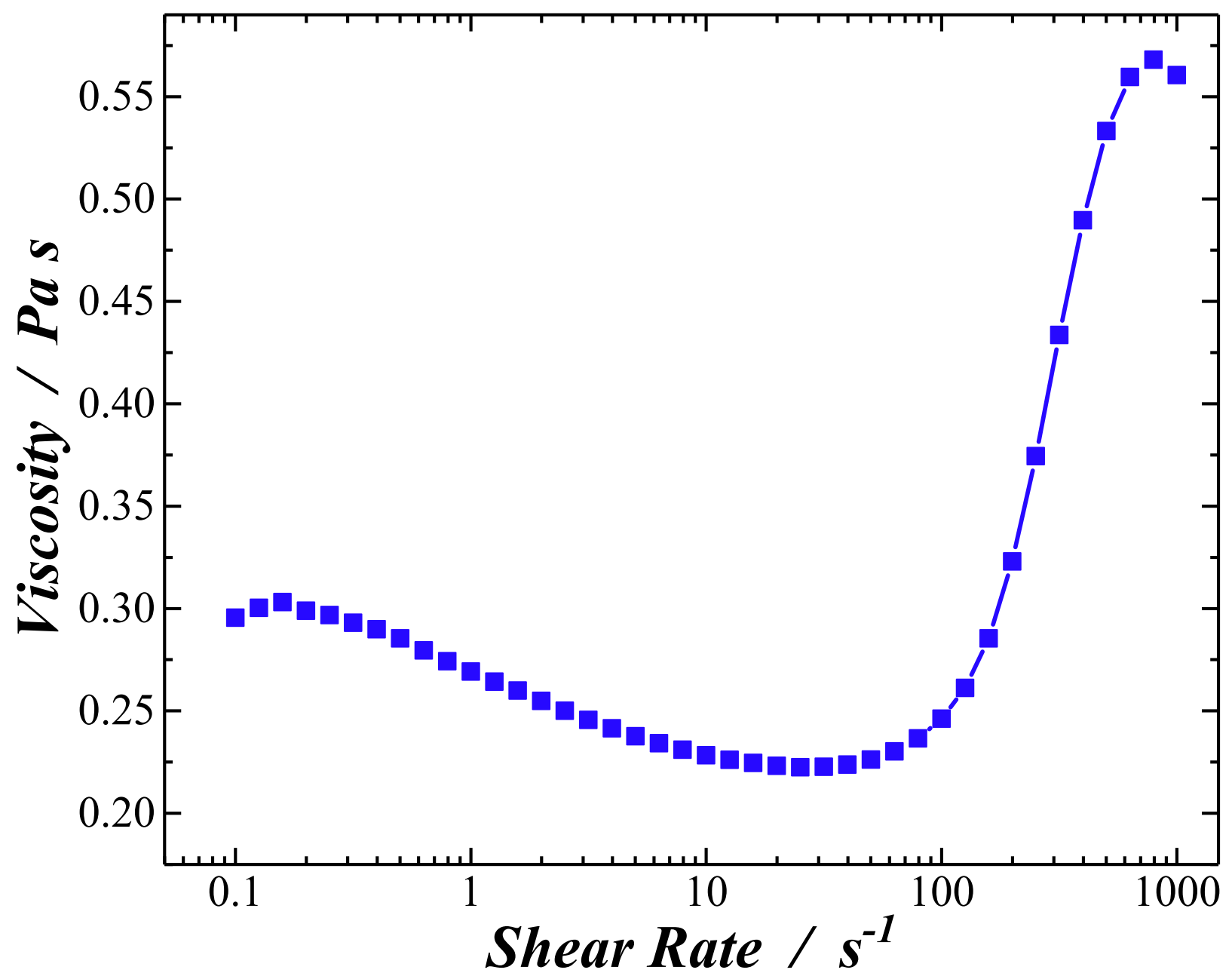

Figure S5. Steady-shear rheological experiments showing the dynamic viscosity of the shearthickening fluid (9 wt\% fumed silica in PEG200) as a function of steady-shear rate at 1\% strain. 



Figure S6. Steady-shear rheological experiments showing the dynamic viscosity of the various core fluids as a function of shear rate (left) and shear time (right; shear rate $=100 \mathrm{~s}^{-1}$ ) at $1 \%$ stain. 

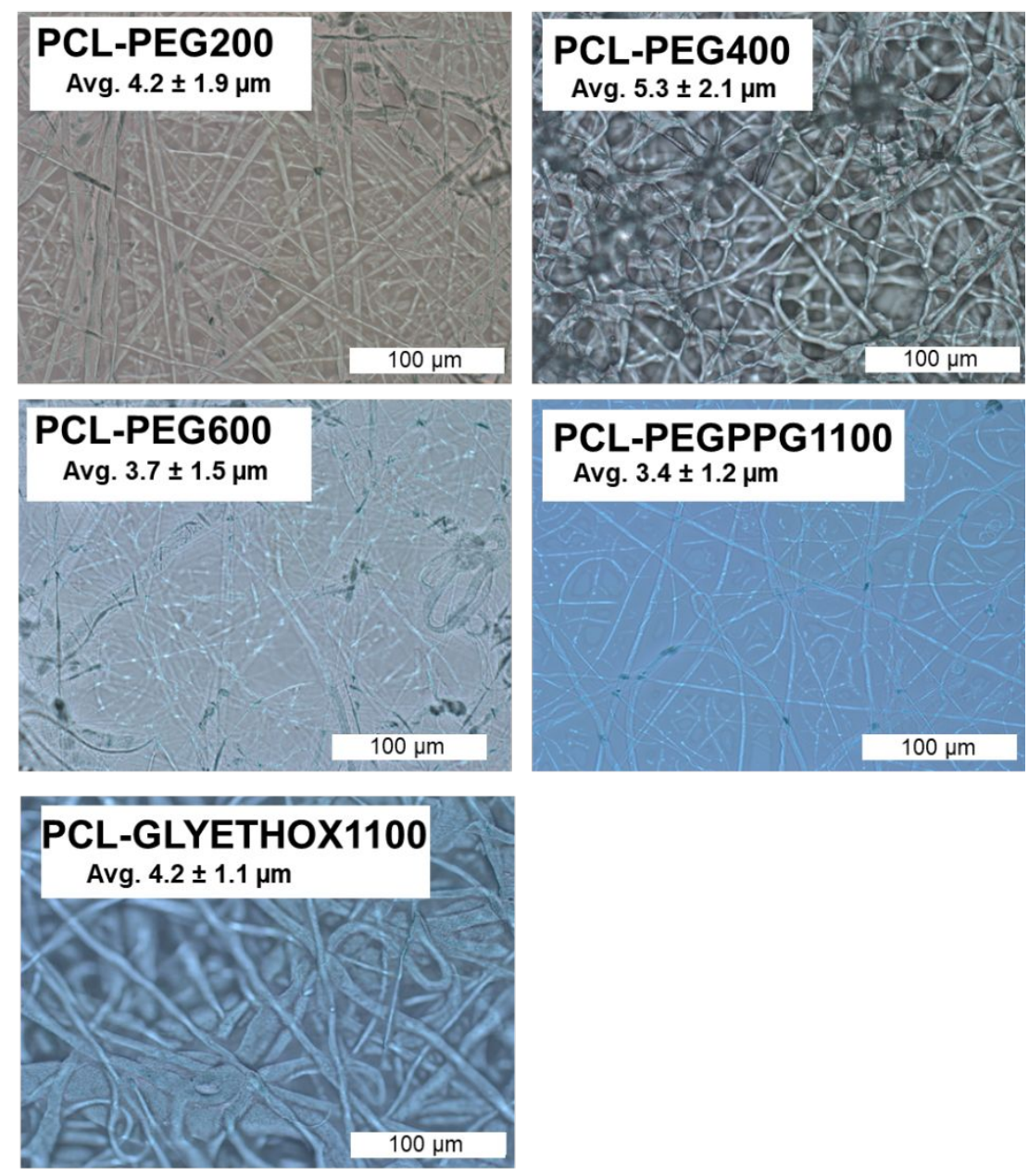

Figure S7. Representative optical microscopy images of core-sheath fibers with composition and average fiber diameter ( \pm 1 standard deviation) shown in insets. Scale bars are $100 \mu \mathrm{m}$. 